\title{
Analytical and bioanalytical science in China
}

\author{
Lihua Zhang • Qiankun Zhuang • Yukui Zhang
}

Published online: 14 February 2011

(C) Springer-Verlag 2011

In the past few years, analytical and bioanalytical science in China has seen rapid development. According to the practical requirements put forward by diverse fields related to human health, such as life science, environmental science, and food safety, much effort has been made to improve the selectivity, sensitivity, speediness, accuracy, and automation of complex sample analysis. Therefore, various novel materials, methods, techniques, and platforms have been developed, and have been successfully applied to the profiling or target analysis of proteomes, metabonomes, natural products, environmental pollutants, food additives, and so forth, and have shown superiority to achieve highefficiency sample preparation, high-resolution separation, and high-sensitivity detection. Furthermore, much attention has been paid to the development of novel techniques to study the interaction, imaging, and location of molecules.

On one hand, Chinese scholars are encouraged to do their best to carry out interdisciplinary research. Especially

Published in the special issue Analytical and Bioanalytical Science in China with Guest Editors Lihua Zhang, Qiankun Zhuang and Yukui Zhang.

\section{Zhang $(\bowtie) \cdot$ Y. Zhang}

National Chromatographic R. \& A. Center,

Key Laboratory of Separation Science for Analytical Chemistry,

Dalian Institute of Chemical Physics,

Chinese Academy of Sciences,

457 Zhongshan Road,

Dalian 116023, China

e-mail: lihuazhang@dicp.ac.cn

\section{Q. Zhuang}

Department of Chemical Science,

National Natural Science Foundation of China,

83 Shuangqing Road,

Beijing 100085, China with the adoption of physical knowledge, much effort has been made to design novel analytical instrumentation to carry out original and innovative research. On the other hand, various analytical and bioanalytical methods have been developed to solve problems proposed by practical applications. Therefore, as a tool subject, analytical and bioanalytical science in China is playing an important role in modern society.

This special issue, with 17 papers, including three reviews, 13 original papers, and one short communication, can only reflect the tip of the huge iceberg of analytical and bioanalytical science in China. We hope that through the publication of this special issue with the contributions of Chinese scholars, the worldwide scientific community will become more and more acquainted with the research being carried out in China.

We are looking forward to your kind comments, suggestions, and cooperation.

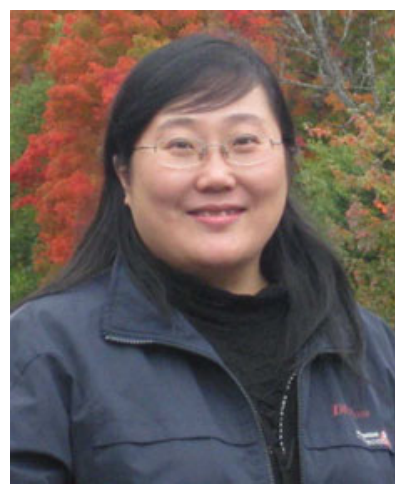

\section{Lihua Zhang}

is a professor at Dalian Institute of Chemical Physics, Chinese Academy of Sciences. Her research and interests focus on the development of new separation and identification methods for both qualitative and quantitative analysis of proteomes, by developing novel sample preparation techniques, multidimensional liquid-phase-based platforms, and coupling with mass spectrometry. 


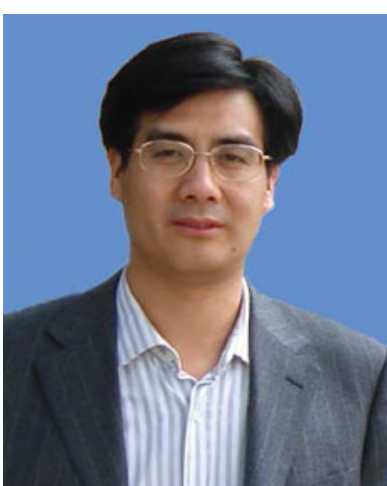

Qiankun Zhuang

is Director of the Analytical Chemistry Division, National Natural Science Foundation of China. He is an expert in electroanalytical chemistry, and is presently strongly involved in promoting the advancement of analytical and bioanalytical science in China

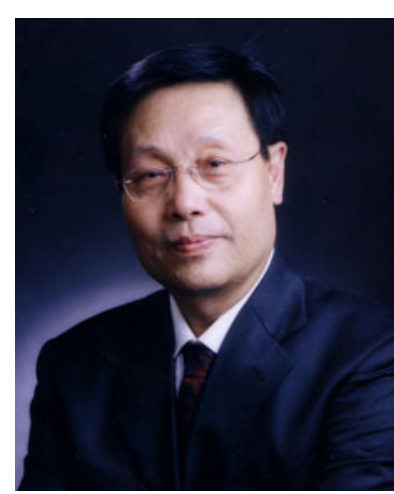

Yukui Zhang

is a member of the Chinese Academy of Sciences and a professor at Dalian Institute of Chemical Physics. His research is focused on the study of the fundamentals of chromatography, column chromatography, and instrumentation for chromatography, as well as applications in the bioanalysis of DNA, bile acids, sugars, and proteins. He is also member of the International Advisory Board of Analytical and Bioanalytical Chemistry. 